\title{
Clinicopathological correlation of tumor-associated macrophages in Ewing sarcoma
}

\author{
Marek Handla ${ }^{a}$, Marketa Hermanova ${ }^{b}$, Sylva Hotarkova ${ }^{b}$, Jiri Jarkovskyc, Peter Mudry ${ }^{d}$, Tetiana Shatokhina ${ }^{b}$, Marcela Vesela ${ }^{b}$, \\ Jaroslav Sterba ${ }^{d}$, Iva Zambo ${ }^{b}$
}

\begin{abstract}
Aims. Tumor-associated macrophages (TAMs) are known markers playing complex roles in tumorigenesis. However, the function of TAMs in a variety of malignancies is not yet fully understood. The aim of this pilot study was to quantify the density of TAMs in Ewing sarcoma and to determine the correlation between TAMs and clinicopathological parameters. Methods. Using immunohistochemistry, the expressions of CD68 and CD163 were examined in 24 tissue samples of Ewing sarcoma of bone. The density of CD68 and CD163-positive TAMs was analyzed quantitatively and semi-quantitatively and statistically correlated with clinical parameters.

Results. CD163-positive TAMs outnumbered CD68-positive cells (median of 130 vs 96, respectively). No statistically significant relatio nship was found between density of CD68-positive cells, clinical parameters or prognosis. However, high levels of CD163-positive TAMs were associated with localized disease $(P=0.008)$. In cases with a higher density of CD163-positive cells, a trend toward longer survival was revealed $(P=0.063)$.

Conclusion. This is the first study that has quantified CD163 expression in TAMs in Ewing sarcoma and showed its possible prognostic value. CD163 was confirmed to be a more specific marker of macrophages than CD68. CD163 is not an exclusive hallmark of M2 macrophages.
\end{abstract}

Key words: tumor associated macrophages, Ewing sarcoma, immunohistochemistry, CD68, CD163, prognosis

Received: August 3, 2017; Accepted: October 26, 2017; Available online: November 21, 2017

https://doi.org/10.5507/bp.2017.049

${ }^{a}$ Faculty of Medicine, Masaryk University, Brno, Czech Republic

${ }^{b} 1^{\text {st }}$ Department of Pathological Anatomy, Faculty of Medicine, Masaryk University, Brno and St. Anne's University Hospital, Brno, Czech Republic

'Institute of Biostatistics and Analyses, Masaryk University, Brno, Czech Republic

${ }^{d}$ Department of Pediatric Oncology, University Hospital Brno and Faculty of Medicine, Masaryk University, Brno, Czech Republic

Corresponding author: Iva Zambo, e-mail: iva.zambo@fnusa.cz

\section{INTRODUCTION}

Ewing sarcoma (ES) represents the second most common primary bone cancer of childhood, adolescence and young adults. ES has a predilection for Caucasians and typically arises in bone marrow, but extra skeletal soft tissues can also be affected ${ }^{1}$. It is an extremely aggressive tumor with a high propensity for local recurrences and distant metastases. At the time of diagnosis, the hematogenous spread to lung and/or bone is detectable in about $25 \%$ of patients. Although the current five-year survival rate is about $70 \%$ representing a significant advance in prognosis in patients with localized disease, the outcome for patients with early relapse or primary disseminated disease remains less than $30 \%\left(\right.$ ref. $\left.^{2}\right)$. Several clinical and pathological factors, such as metastatic spread, histological response to chemotherapy, primary tumor localization, and tumor size and volume, are considered to be predictive of prognosis ${ }^{3,4}$. However, little is known about the molecular markers that could be used for prognostic risk stratification at the time of diagnostic biopsy, thus before chemotherapy administration. Moreover, recent data have shown that factors based on the biological profile could better reflect the malignancy of mesenchymal neoplasias, not only in ES (ref. ${ }^{5,6}$ ).
The existence of a tumor microenvironment and the interaction between stromal and tumor cells is implicated in cancer development and progression ${ }^{7}$. Macrophages play a crucial role in this interaction, having the ability to promote tumor growth, invasion, angiogenesis and metastasis $^{8-11}$. Moreover, these tumor-associated macrophages (TAMs) contribute to tumor relapse following anticancer therapy ${ }^{12}$. Some studies have proven a correlation between large number of TAMs and poor prognosis, advanced grade or stage of various cancers $s^{13-16}$. Other studies have produced contradictory results ${ }^{17-19}$, making this issue highly controversia ${ }^{21}$. The answer may reside in the fact that macrophages are highly plastic and can alter their phenotype flexibly according to local stimuli. Briefly summarized, various subsets of TAMs are recognized with the two extremes of possible differentiation states: the classically activated, proinflammatory M1 macrophages, and the alternatively activated, tumor promoting M2 macrophages ${ }^{22}$. Interferon- $\gamma$ and microbial products polarize macrophages along the M1 pathway. M1 macrophages secrete IL-12, TNF , inducible nitric oxide synthase (iNOS) and reactive oxygen and nitrogen species (ROS, RNS), thus activating the cytotoxic Th1 immunity response and tumor suppression ${ }^{8,23}$. On the other side of the spectrum are M2 macrophages that 
can be differentiated in the presence of IL-4, IL-10, IL13, PGE2 and TGF $\beta$. M2 macrophages produce IL-10, IL-1 receptor antagonist, CC ligand 22 (CCL22), matrix metalloproteinases and scavenger and mannose receptor. This way promotes tissue remodeling and angiogenesis, which supports tumor progression ${ }^{24,25}$. In the past, M2 macrophages were almost exclusively considered to be responsible for tumor promoting effects but accumulating evidence have suggested that it was an oversimplification. TAMs are composed of multiple macrophage populations with overlapping features that depend on the mix of signals in their direct microenvironment ${ }^{26,27}$.

Currently, the role of TAMs in ES is not fully understood. To the best of our knowledge, there has been only one published study focused on the relationship between the number of CD68-positive cells and clinical outcome in ES patients ${ }^{15}$.

In this study, the expressions of CD68 and CD163 in ES tissue samples were immunohistochemically examined. The densities of TAMs were analyzed quantitatively and semi-quantitatively and number of infiltrating TAMs as well as expression levels of TAMs were correlated with clinicopathological parameters and prognosis.

\section{MATERIALS AND METHODS}

\section{Study group and tissue specimens}

The study sample consisted of 24 patients with primary ES who had undergone surgical intervention at the St. Anne's University Hospital between 2006 and 2012. Follow-up data were available until February 2015. All tumor tissue samples were obtained prior to administration chemotherapy or radiotherapy and were bioptically confirmed by pathologists who had special expertise in sarcomas. The final diagnosis of ES was supported by detection of EWSRl gene rearrangement. The corresponding formalin-fixed, paraffin-embedded blocks were obtained from the archive of the $1^{\text {st }}$ Department of Pathological Anatomy of the St. Anne's University Hospital in Brno, Czech Republic. Disease staging was performed according to American Joint Committee on Cancer (AJCC) staging system.

The study was approved by the Ethics Committee of the University Hospital Brno. All procedures to obtain human tumor tissue samples and follow-up information were in accordance with the ethical standards of the institutional and national research committee, with the legislation of the Czech Republic and with the 1964 Helsinki declaration (revised in 2013). Informed consent was obtained from all individual patients or their guardians. The individual patient cannot be identified from any material in the manuscript.

\section{Immunohistochemistry}

Immunohistochemical analysis of CD68 and CD163 expression was performed on 4- $\mu$ m thick tissue sections mounted on positively charged glass slides. Sections were deparaffinized with xylene and rehydrated through a graded series of ethanol. Antigen retrieval was performed in the calibrated pressure chamber Pascal (DAKO, Glostrup, Denmark) by heating the sections in Tris/EDTA buffer (Dako) at $\mathrm{pH} 9.0$ at $117{ }^{\circ} \mathrm{C}$ for $1 \mathrm{~min}$. Endogenous peroxidase activity was quenched in $3 \%$ hydrogen peroxide in methanol for $20 \mathrm{~min}$. The slides were subsequently incubated at room temperature for $60 \mathrm{~min}$ with a mouse monoclonal antibody against CD68 (clone KP1, dilution 1:100, Dako, Glostrup, Denmark) or a mouse monoclonal antibody against CD163 (clone 2G12, dilution 5:100, Abcam, Cambridge, UK). Tissue sections were then incubated with anti-mouse secondary antibody. For CD163, a streptavidin-biotin peroxidase detection system was used in accordance with manufacturer's instructions (Vesctastain Ellite ABC Kit, Vector, Burlingame, California, USA). For CD68, two-step detection system without avidin or biotin was applied (EnVision+ Dual Link system-HRP, DAKO). All slides were immersed in 3,3'-diaminobenzidine (DAB, Dako) and counterstained with Gill's hematoxylin. Negative controls were prepared by incubating samples without primary antibody. For CD163 and CD68, Kupffer cells in liver and normal tissues of appendix were used as external positive controls, respectively.

All immunostained slides were evaluated by two independent observers ( $\mathrm{MH}$ and $\mathrm{SH}$ ) without any knowledge of the clinicopathological information.

\section{Evaluation of immunostaining}

The representative fields of immunostaining were chosen at low magnification. The density of CD68 and CD163 positive cells were quantitatively assessed by using

Table 1. Clinicopathological characteristics of patients with ES $(n=24)$.

\begin{tabular}{lcc}
\hline Variables & & Median $($ range $) / \mathrm{n}(\%)^{1}$ \\
\hline Age & years & $12.5(2.0-27.0)$ \\
Gender & Male & $13(54.2 \%)$ \\
Stage & Female & $11(45.8 \%)$ \\
& $2 \mathrm{a}$ & $5(20.8 \%)$ \\
& $2 \mathrm{~b}$ & $6(25.0 \%)$ \\
M status & 3 & $3(12.5 \%)$ \\
& 4 & $10(41.7 \%)$ \\
R0 resection & 0 & $14(58.3 \%)$ \\
& 1 & $10(41.7 \%)$ \\
CR & no & $4(16.7 \%)$ \\
Follow-up length & yes & $20(83.3 \%)$ \\
CD68 & no & $8(33.3 \%)$ \\
CD68 level & yenths & $16(66.7 \%)$ \\
& 1 & $54(11-109)$ \\
CD163 & 2 & $96(33-257)$ \\
CD163 level & 3 & $7(29.2 \%)$ \\
& numbers & $13(29.2 \%)$ \\
& 1 & $5(41.7 \%)$ \\
& 2 & $10(41.7 \%)$ \\
\end{tabular}

${ }^{1}$ median (minimum - maximum)/count (percentage) 
Table 2. Relationship between CD68 expression and clinicopathological variables of ES.

\begin{tabular}{|c|c|c|c|c|c|c|c|}
\hline \multirow{2}{*}{$n=24$} & & \multirow{2}{*}{$\mathrm{CD} 68^{1}$} & \multirow{2}{*}{$P^{2}$} & \multicolumn{3}{|c|}{ CD68 level $^{3}$} & \multirow{2}{*}{$P^{4}$} \\
\hline & & & & 1 & 2 & 3 & \\
\hline Age & & 0.313 & 0.137 & $7.0(2-19)$ & $13.0(7-18)$ & $13.0(4-27)$ & 0.249 \\
\hline \multirow[t]{2}{*}{ Gender } & Male & $77.0(49-201)$ & 0.562 & $3(27.3 \%)$ & $2(18.2 \%)$ & $6(54.5 \%)$ & 0.434 \\
\hline & Female & $135.0(33-257)$ & & $4(30.8 \%)$ & $5(38.5 \%)$ & $4(0.0 \%)$ & \\
\hline \multirow[t]{2}{*}{ M status } & 0 & $90.5(49-201)$ & 0.907 & $4(28.6 \%)$ & $4(28.6 \%)$ & $6(42.9 \%)$ & 0.990 \\
\hline & 1 & $101.0(33-257)$ & & $3(30.0 \%)$ & $3(30.0 \%)$ & $4(40.0 \%)$ & \\
\hline \multirow[t]{2}{*}{ R0 resection } & yes & $130.5(65-201)$ & 0.278 & $0(0.0 \%)$ & $2(50.0 \%)$ & $2(50.0 \%)$ & 0.198 \\
\hline & no & $95.5(33-257)$ & & $7(35.0 \%)$ & $5(25.0 \%)$ & $8(40.0 \%)$ & \\
\hline \multirow[t]{2}{*}{$\mathrm{CR}$} & no & $122.5(57-257)$ & 0.327 & $1(12.5 \%)$ & $3(37.5 \%)$ & $4(50.0 \%)$ & 0.409 \\
\hline & yes & $73.0(33-201)$ & & $6(37.5 \%)$ & $4(25.0 \%)$ & $6(37.5 \%)$ & \\
\hline
\end{tabular}

${ }^{1}$ Spearman correlation coefficient/median (minimum-maximum)

${ }^{2}$ Significance of Spearman correlation coefficient/Kruskal-Wallis test

${ }^{3}$ absolute (relative frequency)/median (minimum-maximum)

${ }^{4}$ ML-chi square test/Kruskal-Wallis test

Table 3. Relationship between CD163 expression and clinicopathological variables of ES.

\begin{tabular}{|c|c|c|c|c|c|c|c|}
\hline \multirow{2}{*}{$\mathrm{n}=24$} & & \multirow{2}{*}{$\mathrm{CD} 163^{1}$} & \multirow{2}{*}{$P^{2}$} & \multicolumn{3}{|c|}{ CD163 level $^{3}$} & \multirow{2}{*}{$P^{4}$} \\
\hline & & & & 1 & 2 & 3 & \\
\hline Age & & 0.139 & 0.516 & $10(2-19)$ & $13,5(5-27)$ & $12(3-18)$ & 0.666 \\
\hline \multirow{2}{*}{ Gender } & Male & $127.0(58-208)$ & \multirow{2}{*}{0.369} & $3(27.3 \%)$ & $4(36.4 \%)$ & $4(36.4 \%)$ & \multirow{2}{*}{0.760} \\
\hline & Female & $133.0(61-355)$ & & $2(15.4 \%)$ & $6(46.2 \%)$ & $5(38.5 \%)$ & \\
\hline \multirow{2}{*}{ M status } & 0 & $158.5(89-314)$ & \multirow{2}{*}{0.008} & $0(0.0 \%)$ & $6(42.9 \%)$ & $8(57.1 \%)$ & \multirow{2}{*}{0.002} \\
\hline & 1 & $83.0(58-355)$ & & $5(50.0 \%)$ & $4(40.0 \%)$ & $1(10.0 \%)$ & \\
\hline \multirow{2}{*}{ R0 resection } & yes & $142.5(131-189)$ & \multirow{2}{*}{0.278} & $0(0.0 \%)$ & $2(50.0 \%)$ & $2(50.0 \%)$ & \multirow{2}{*}{0.353} \\
\hline & no & $123.5(58-355)$ & & $5(25.0 \%)$ & $8(40.0 \%)$ & $7(35.0 \%)$ & \\
\hline \multirow{2}{*}{ CR } & no & $109.0(62-178)$ & \multirow{2}{*}{0.126} & $2(25.0 \%)$ & $5(62.5 \%)$ & $1(12.5 \%)$ & \multirow{2}{*}{0.159} \\
\hline & yes & $144.5(58-355)$ & & $3(18.8 \%)$ & $5(31.3 \%)$ & $8(50.0 \%)$ & \\
\hline
\end{tabular}

${ }^{1}$ Spearman correlation coefficient/median (minimum-maximum)

${ }^{2}$ Significance of Spearman correlation coefficient/Kruskal-Wallis test

${ }^{3}$ absolute (relative frequency)/median (minimum-maximum)

${ }^{4}$ ML-chi square test/Kruskal-Wallis test

NIS-Elements Software. The macrophages were counted in an image with an area of $1 \mathrm{~mm}^{2}$, which was created from 20 different visual fields $(0.2 \mathrm{~mm} \times 0.25 \mathrm{~mm})$. The cases were then stratified into three levels according to the number of TAMs. The proportion of CD68 positive cells was scored as follows: $1,<60$ positive cells; $2,61-130$ positive cells; and 3,>131 positive cells. Levels of CD163 positive cells were specified as: $1,<80$ positive cells; 2 , 81-140 positive cells; and 3,>140 positive cells.

\section{Statistical analysis}

Standard descriptive statistics included absolute and relative frequencies for categorical variables and median with minimum-maximum range for continuous variables. Relations between continuous variables were described using Spearman correlation coefficient. Statistical significance between groups was tested using the maximum likelihood chi-square test for categorical variables and Kruskal-Wallis test for continuous variables. Survival of patients was visualised using the Kaplan-Meier curve; effect of patient characteristics on survival was analysed using the Cox proportional hazards model and described using hazard ratios and $95 \%$ confidence interval (CI).
The SPSS 23.0.0 (IBM Corporation, 2015) was used for the analyses.

\section{RESULTS}

The characteristics of the study cohort are listed in Table 1 . The study sample consisted of 13 (54.2\%) males and $11(45.8 \%)$ females. The median age was 12.5 years (range 2.0 - 27.0). All patients completed the standard therapeutic regimen. The follow-up periods ranged from 11 to 109 months (median 54.0 months). Localized disease was noted in 14 patients $(58.3 \%)$. At the time of diagnosis, ten patients (41.7\%) had AJCC Stage 4, which refers to distant metastatic disease at onset. Total of $20(83.3 \%)$ patients underwent surgery and at least one complete remission occurred in $16(66.7 \%)$ patients. At the last censored time, 17 (70.8\%) patients were alive. Of these 17 survivors, 14 remained with no evidence of disease and 3 went to the remission after receiving a treatment for relapse. Total of 7 (29.2\%) patients succumbed to disease progression.

Using immunohistochemistry, expressions of CD68 

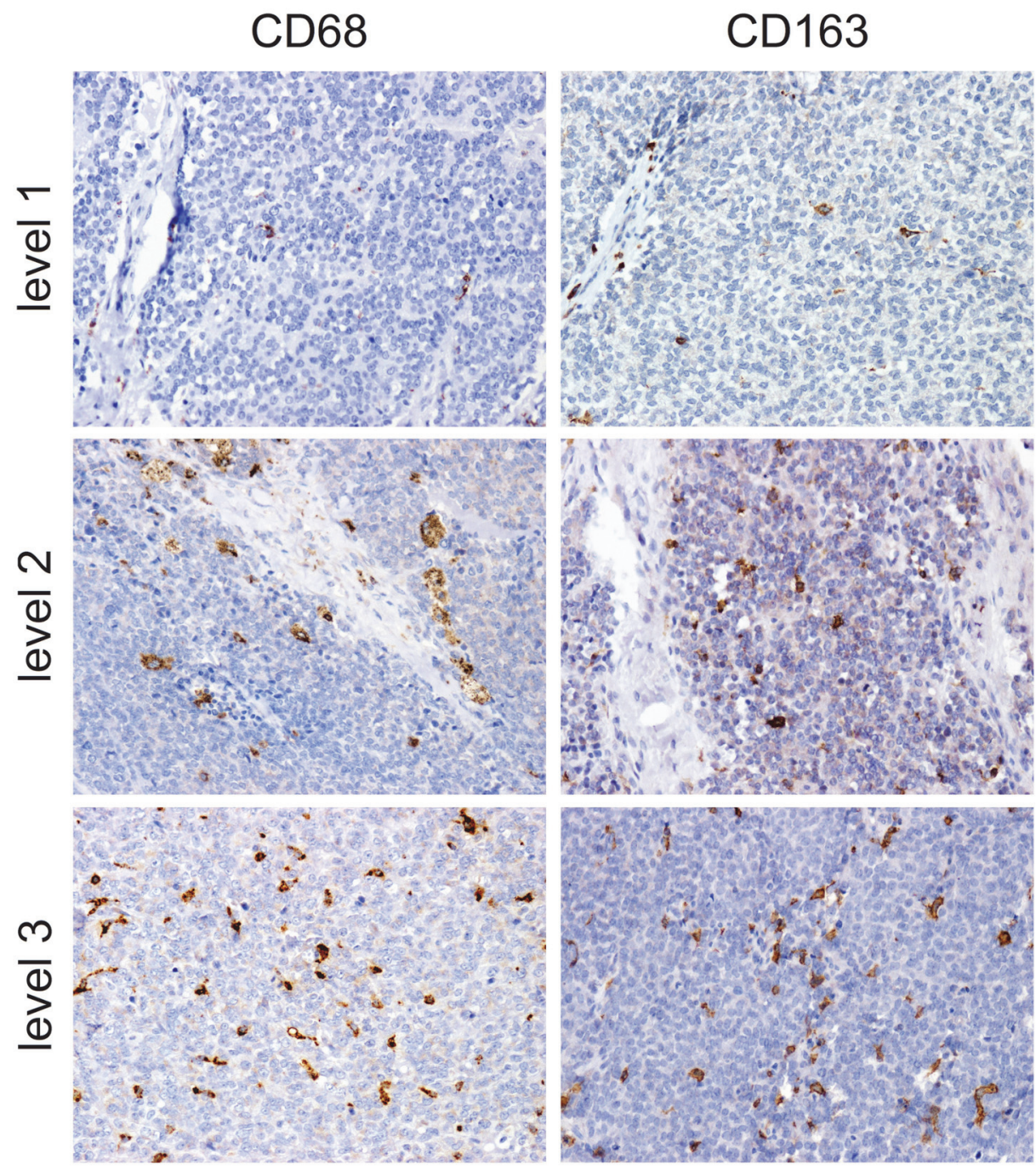

Fig. 1. Expression of CD68 and CD163 in Ewing sarcoma.

The proportion of CD68 positive cells was scored as follows: $1,<60$ positive cells; 2, 61-130 positive cells; and 3,>131 positive cells. Expression levels of CD163 were specified as: $1,<80$ positive cells; 2 , $81-140$ positive cells; and 3,>140 positive cells. Original magnification $\times 200$

and CD163 were evaluated and quantitatively and semiqauntitatively analyzed. Representative immunostainings of infiltrating CD68 and CD163-positive cells are shown in Fig. 1.

Overall, CD163-positive TAMs outnumbered CD68positive cells (median of 130 vs 96, respectively). Approximately $71 \%$ and $79 \%$ of cases displayed a modest to massive infiltration of CD68 or CD163-positive cells (levels 2 and 3), respectively. The density or levels of CD68-positive cells were not significantly associated with clinical parameters or prognosis. However, the number of CD163-positive TAMs was correlated with M0 status $(P=0.008)$. Similar results were obtained by comparing the levels of CD163 expression with M status: cases with high-level of CD163 expression (level 3) were significantly associated with localized disease $(P=0.002)$. Relationships between CD68 and CD163 expression and clinicopathological parameters are shown in Table 2 and 3. Univariate Cox proportional hazard regression analysis revealed a strong trend toward longer survival in relation to a higher density of CD163-positive TAMs, with Hazard Ratio 0.99 (95\% CI, 0.97-1.00), however these results did not reach statistical significance ( $P=0.063$, Fig. 2$)$. Results of univariate analyses for overall survival are listed in Table 4 and Fig. 2.

\section{DISCUSSION}

ES is characterized by highly aggressive behavior. Since the introduction of multimodal treatment relevant advances have been achieved for prognosis in patients with localized disease. Nevertheless, the outcome for patients with early relapse or primary metastatic disease remains poor ${ }^{2}$. Currently, prognostic factors in ES are mostly derived from clinical parameters. However, molecular markers usable for prognostic risk stratification at the time of diagnosis have not yet been established.

TAMs represent a group of stromal cells that play an important role in oncogenesis and tumor progression ${ }^{28}$. 


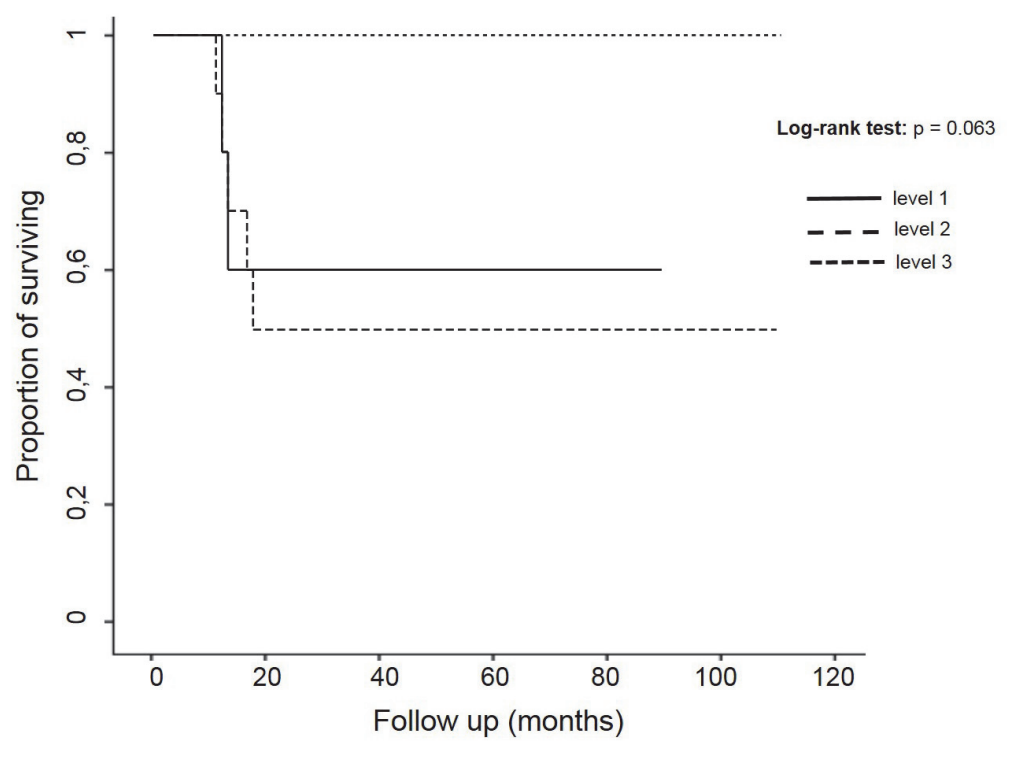

Fig. 2. Overall survival according to levels of CD163.

Due to the heterogeneity of the macrophage population, their role in the tumor microenvironment is very complex $^{22-27}$. TAMs can develop a continuum of polarization states with the tumor-suppressing M1 macrophages lying on one end and the tumor-promoting M2 macrophages on the other. The proportional representation and localization of the different phenotypes of TAMs in the tumor tissue determine the overall influence of the macrophage population in a given neoplasia ${ }^{26,27}$. For the patient, the effect of TAMs can therefore be either beneficial or adverse $^{29,30}$. The phenotypic diversity of the TAMs is probably the main reason for the unclear picture of their effect on tumor progression. There have been studies that support the tumor-promoting roles of the TAMs (ref. ${ }^{13-16}$ ). On the other hand, some studies have identified the effect of TAMs as tumor-suppressing ${ }^{17-20}$.

This heterogeneity of the results is caused by the inability to clearly distinguish between TAM subtypes. Various studies have used different markers for the identification of TAMs, the most common being CD68 and CD163 (ref. ${ }^{24}$ ). CD68 is considered to be a pan-macrophage marker and CD163 was reported to show promising signs of being a somewhat specific marker of M2 macrophages ${ }^{31,32}$. However, our study suggests these assumptions may not be generally applicable. While CD68 is a marker commonly used as a macrophage marker in routine histological practice, it has been reported to be relatively non-specific, with positive staining in a wide variety of haematopoietic and non-haematopoietic cell types. This is in concordance with the fact, that CD68 is a lysosome-associated rather than histiocytic marker ${ }^{33-35}$. As demonstrated in our study CD68 cannot be considered a reliable marker for TAMs due to its low sensitivity.

CD163, however, appears to be a better alternative to CD68. The expression of CD163 in lymphomas, car-
Table 4. Overall survival according to CD68 and CD163.

\begin{tabular}{|c|c|c|c|}
\hline & & $\mathrm{HR}(95 \% \mathrm{CI})$ & $P$ \\
\hline CD68 & & $1.01(0.99-1.02)$ & NS \\
\hline \multirow{3}{*}{ CD68 level } & 1 & 1 & \\
\hline & 2 & $2.05(0.18-22.63)$ & NS \\
\hline & 3 & $3.39(0.38-30.38)$ & NS \\
\hline CD163 & & $0.99(0.97-1.00)$ & 0.063 \\
\hline \multirow[t]{3}{*}{ CD163 level } & 1 & 1 & \\
\hline & 2 & $1.26(0.25-6.51)$ & NS \\
\hline & 3 & - & \\
\hline
\end{tabular}

$H R$, hazard ratio; $C I$, confidence interval; $N S$, not significant

cinomas and sarcomas has been shown to be highly specific and largely restricted to the monocyte/macrophage lineage ${ }^{36}$. Furthermore, CD163-positive macrophages may in some cases outnumber CD68-positive cells ${ }^{37,41}$ as indicated by our study. Many reports have used CD163 for identification of M2 macrophages ${ }^{14,16,17,31,32}$. However some studies have suggested, as well as our results, that CD163, being a good marker for TAMs in general, cannot be considered an exclusive marker of M2 macrophages when used on its own ${ }^{37,38}$. Thus, using CD163, we are only able to identify the TAMs but unable to distinguish between the different phenotypes.

To date, few published studies have focused on TAMs in human sarcoma tissues. In a cohort of 53 high-grade osteosarcomas, the number of CD14-positive TAMs was associated with reduced metastasis and improved survival, whereas the phenotype CD14/CD163 did not show this relationship ${ }^{18}$. The significance of CD68 and CD163 positive cells was also investigated in 149 samples of leiomyosarcomas. In cases of nongynecologic localization, CD163 or CD68 staining was associated with a 
significantly poorer disease-specific survival, however, no correlation between macrophage infiltration and survival was revealed in leiomyosarcomas arising from the gynecologic tract ${ }^{42}$. Analysis of 47 cases of gastrointestinal stromal tumor showed a dominance of the CD14/CD163positive subset of TAMs within a macrophage population and these cells were more frequently found in metastatic lesions compared to primary tumors ${ }^{43}$. One study so far has examined the macrophage infiltration in ES (ref. ${ }^{15}$ ). In total of 41 samples of ES, Fujiwara and co-workers analyzed the prognostic impact of CD68 expression in infiltrating macrophages. Our study did not confirm reported findings that higher levels of CD68-positive macrophages correlate with poorer overall survival, as we did not find any statistically significant relationship between CD68positive cells, disease stage or survival. For the first time, we have quantified the expression of CD163 in ES and analyzed the impact of CD163-positive TAMs in relation to clinical variables and prognosis. In our study, CD163positive TAMs correlated with lower stage, in other words localized disease of ES. Moreover, a strong trend toward longer survival was evident in relation to the density of CD163-positive cells.

\section{CONCLUSION}

In conclusion, CD163 represents a sensitive marker for macrophages, although it lacks specificity for the M2 phenotype. Our preliminary study revealed an association between CD163 expression and lower stage ES. The findings suggest the usefulness of future detailed analysis of TAMs in a larger cohort of patients with ES.

Acknowledgement: This study was supported by project no. MUNI/FR/0377/2014 of Masaryk University, Brno, Czech Republic.

Author contribution: All authors contributed equally to this manuscript.

Conflict of interest statement: The authors state that there is no conflict of interest regarding the publication of this article.

\section{REFERENCES}

1. Alava E, Lessnick SL, Sorensen PH. Ewing sarcoma. In: Fletcher CDM, Bridge JA, Hogendoorn PCW, Mertens F, editors. WHO classification of tumours of soft tissue and bone, $4^{\text {th }}$ ed, Lyon: IARC; 2013. p. 306339.

2. Duchman KR, Gao Z, Miller BJ. Prognostic factors for survival in patients with Ewing's sarcoma using the surveillance, epidemiology, and end results (SEER) program database. Cancer Epidemiology 2015;39:189-95. doi: 10.1016/j.canep.2015.05.001

3. Cotterill SJ, Ahrens S, Paulussen M, Jürgens HF, Voute PA, Gadner $\mathrm{H}$, Craft AW. Prognostic factors in Ewing's tumor of bone: analysis of 975 patients from the European Intergroup Cooperative Ewing's Sarcoma Study group. J Clin Oncol 2000;18(17):3108-14.

4. Esiashvili N, Goodman M, Marcus RB. Changes in Incidence and Survival of Ewing Sarcoma Patients Over the Past 3 Decades: Surveillance Epidemiology and End Results Data. J Pediatr Hematol Oncol 2008;30(6):425-30. doi:10.1097/MPH.0b013e31816e22f3

5. Huang HY, Illei PB, Zhao Z, Mazumdar M, Huvos AG, Healey JH,
Wexler LH, Gorlick R, Meyers P, Landanyi M. Ewing sarcomas with p53 mutation or p16/p14ARF homozygous deletion: a highly lethal subset associated with poor chemoresponse, J Clin Oncol 2005:23(3):548-58.

6. Futamura N, Nishida Y, Urakawa H, Kozawa E, Ikuta K, Hamada S, Ishiguro N. EMMPRIN co-expressed with matrix metalloproteinases predicts poor prognosis in patients with osteosarcoma. Tumour Biol 2014;35(6):5159-65. doi: 10.1007/s13277-014-1668-8

7. Pietras K, Östman A. Hallmarks of cancer: Interactions with the tumor stroma. Exp Cell Res 2010;316(8):1324-31. doi:10.1016/j. yexcr.2010.02.045

8. Qian BZ, Pollard JW. Macrophage diversity enhances tumor progression and metastasis. Cell 2010;141(1):39-51. doi:10.1016/j. cell.2010.03.014

9. Gocheva V, Wang HW, Gadea BB, Shree T, Hunter KE, Garfall AL, Berman T, Joyce JA. IL-4 induces cathepsin protease activity in tumor-associated macrophages to promote cancer growth and invasion. Genes Dev 2010;24(3):241-55. doi:10.1101/gad.1874010

10. Kessenbrock K, Plaks V, Werb Z. Matrix metalloproteinases: Regulators of the Tumor Microenvironment. Cell 2010;141(1):5267. doi:10.1016/j.cell.2010.03.015

11. Lewis CE, Pollard JW. Distinct role of macrophages in different tumor microenvironments. Cancer Res 2006;66(2):605-12.

12. De Palma $M$, Lewis CE. Macrophage regulation of tumor responses to anticancer therapies. Cancer Cell 2013;23(3):277-86. doi:10.1016/j. ccr.2013.02.013

13. Shabo I, Olsson H, Sun XF, Svanvik J. Expression of the macrophage antigen CD163 in rectal cancer cells is associated with early local recurrence and reduced survival time. Int J Cancer 2009;125:182631. doi: $10.1002 / \mathrm{ijc} .24506$

14. Kawamura K, Konohara Y, Takaishi K, Katabuchi H, Takeya M. Detection of M2 macrophages and colony-stimulating factor 1 expression in serous and mucinous ovarian epithelial tumors. Pathol Int 2009;59(5):300-5. doi: 10.1111/j.1440-1827.2009.02369.x

15. Fujiwara T, Fukushi J, Yamamoto S, Matsumoto Y, Setsu N, Oda Y, Yamada H, Okada S, Watari K, Ono M, Kuwano M, Kamura S, lida K, Okada Y, Koga M, Iwamoto Y. Macrophage Infiltration Predicts a Poor Prognosis for Human Ewing Sarcoma. Am J Pathol 2011;179(3):115770. doi:10.1016/j.ajpath.2011.05.034

16. Kurahara $H$, Shinchi H, Mataki Y, Maemura K, Noma H, Kubo F, Sakoda M, Ueno S, Natsugoe S, Takao S. Significance of M2-Polarized Tumor-Associated Macrophage in Pancreatic Cancer. J Surg Res 2011;167(2):e211-e219. doi:10.1016/j.jss.2009.05.026

17. Ma J, Liu L, Che G, Yu N, Dai F, You Z. The M1 form of tumor-associated macrophages in non-small cell lung cancer is positively associated with survival time. BMC Cancer 2010;10:112. doi: 10.1186/14712407-10-112

18. Buddingh EP, Kuijjer ML, Duim RA, Bürger $H$, Agelopoulos $K$, Myklebost O, Serra M, Mertens F, Hogendoorn PC, Lankester AC, Cleton-Jansen AM. Tumor-infiltrating macrophages are associated with metastasis suppression in high-grade osteosarcoma: a rationale for treatment with macrophage activating agents. Clin Cancer Res 2011;17(8):2110-19. doi: 10.1158/1078-0432.CCR-10-2047

19. Edin S, Wikberg ML, Dahlin AM, Rutegård J, Öberg $\AA$, Oldenborg PA, Palmqvist R. The distribution of macrophages with a M1 or M2 phenotype in relation to prognosis and the molecular characteristics of colorectal cancer. PLoS One 2012;7(10):e47045. doi: 10.1371/journal. pone.0047045

20. Ohri CM, Shikotra A, Green RH, Waller DA, Bradding P. Macrophages within NSCLC tumour islets are predominantly of a cytotoxic M1 phenotype associated with extended survival. Eur Respir J 2009;33(1):118-26. doi:10.1183/09031936.00065708

21. Zhang QW, Liu L, Gong CY, Shi HS, Zeng YH, Wang XZ, Zhao YW, Wei YQ. Prognostic significance of tumor-associated macrophages in solid tumor: a meta-analysis of the literature. PloS One 2012;17(12):e50946. doi:10.1371/journal.pone.0050946

22. Mantovani A, Sozzani S, Locati M, Allavena P, Sica A. Macrophage polarization: tumor-associated macrophages as a paradigm for polarized M2 mononuclear phagocytes. Trends Immunol 2002;23(11):549-55. doi: 10.1016/S1471-4906(02)02302-5

23. Mantovani A, Sica A. Macrophages, innate immunity and cancer: balance, tolerance, and diversity. Curr Opin Immunol 2010;22(2):231-7. doi:10.1016/j.coi.2010.01.009

24. Heusinkveld M, Van Der Burg SH. Identification and manipulation 
of tumor associated macrophages in human cancers. J Transl Med 2011;9:216. doi: 10.1186/1479-5876-9-216

25. Gordon S, Martinez FO. Alternative Activation of Macrophages: Mechanism and Functions. Immunity 2010;32(5):593-604. doi:10.1016/j.immuni.2010.05.007

26. Mosser DM and Edwards JP. Exploring the full spectrum of macrophage activation. Nat Rev Immunol 2008;8(12):958-69.

27. Schouppe E, De Baetselier P, Van Ginderachter JA, Sarukhan A. Instruction of myeloid cells by the tumor microenvironment: open questions on the dynamics and plasticity of different tumor-associated myeloid cell populations. Oncoimmunology 2012;1(2):1135-45 doi:10.4161/onci.21566

28. Balkwill F, Mantovani A. Inflammation and cancer: back to Virchow?. The Lancet 2001;357(9255):539-45. doi:10.1016/S01406736(00)04046-0

29. Hao NB, Mlü MH, Fan YH, Cao YL, Zhang ZR. Macrophages in tumor microenvironments and the progression of tumors. Clin Dev Immunol 2012;2012:948098. doi: 10.1155/2012/948098

30. Quatromoni JG, Eruslanov E. Tumor-associated macrophages: function, phenotype, and link to prognosis in human lung cancer. Am J Transl Res 2012:4(4):376-89.

31. Komohara Y, Hirahara J, Horikawa T, Kawamura K, Kiyota E, Sakashita $\mathrm{N}$, Araki N, Takeya M. AM-3K, an anti-macrophage antibody, recognizes CD163, a molecule associated with an anti-inflammatory macrophage phenotype. J Histochem Cytochem 2006;54(7):763-71. doi:10.1369/jhc.5A6871.2006

32. Komohara Y, Ohnishi K, Kuratsu J, Takeya M. Possible involvement of the M2 anti-inflammatory macrophage phenotype in growth of human gliomas. J Pathol 2008;216(1):15-24. doi:10.1002/path.2370

33. Gloghini A, Rizzo A, Zanette I, Canal B, Rupolo G, Bassi P, Carbone A $\mathrm{KP} 1 / \mathrm{CD} 68$ expression in malignant neoplasms including lymphomas, sarcomas, and carcinomas. Am J Clin Pathol 1995;103(4):42531.

34. Tsang WY, Chan JK. KP1 (CD68) staining of granular cell neoplasms: is KP1 a marker for lysosomes rather than the histiocytic lineage? Histopathology 1992;21(1):84-6.

35. Gottfried E, Kunz-Schughart LA, Weber A, Rehli M, Peuker A, Müller A, Kastenberger M, Brockhoff G, Andreesen R, Kreutz M. Expression of
CD68 in non-myeloid cell types. Scand J Immunol 2008;67(5):453-63. doi: 10.1111/j.1365-3083.2008.02091.x

36. Nguyen TT, Schwartz EJ, West RB, Warnke RA, Arber DA, Natkuman Y. Expression of CD163 (hemoglobin scavenger receptor) in normal tissues, lymphomas, carcinomas, and sarcomas is largely restricted to the monocyte/macrophage lineage. Am J Surg Pathol 2005;29(5):617-24.

37. Barros MH, Hauck F, Dreyer JH, Kempkes B, Niedobitek G. Macrophage polarisation: an immunohistochemical approach for identifying M1 and M2 macrophages. PloS One 2013;8(11):e80908. doi:10.1371/journal.pone.0080908

38. Harris JA, Jain S, Ren Q, Zarineh A, Liu C, Ibrahim S. CD163 versus CD68 in tumor associated macrophages of classical Hodgkin lymphoma. Diagn Pathol 2012;7:12. doi: 10.1186/1746-1596-7-12

39. Nagorsen D, Voigt S, Berg E, Stein H, Thiel E, Loddenkemper C. Tumor-infiltrating macrophages and dendritic cells in human colorectal cancer: relation to local regulatory $T$ cells, systemic T-cell response against tumor-associated antigens and survival. J Transl Med 2007:5:62. doi:10.1186/1479-5876-5-62

40. Azambuja D, Natkunam Y, Biasoli I, Lossos IS, Anderson MW, Morais JC, Spector M. Lack of association of tumor-associated macrophages with clinical outcome in patients with classical Hodgkin's lymphoma. Ann Oncol 2011;23(3):736-42 doi:10.1093/annonc/mdr157

41. Kong LQ, Zhu XD, Xu HX, Zhang JB, Lu L, Wang WQ, Zhang QB, Wu WZ, Wang L, Fan J, Tang ZY, Sun HC. The clinical significance of the CD163+ and CD68+ macrophages in patients with hepatocellular carcinoma. PLoS One 2013;8(3):e59771. doi: 10.1371/journal. pone.0059771

42. Lee $\mathrm{CH}$, Espinosa I, Vrijaldenhoven $\mathrm{S}$, Subramanian S, Montgomery KD, Zhu S, Marinelli RJ, Peterse JL, Poulin N, Nielsen TO, West RB, Gilks $C B$, van de Rijn M. Prognostic significance of macrophage infiltration in leiomyosarcomas. Clin Cancer Res 2008;14(5):1423-30. doi: 10.1158/1078-0432.CCR-07-1712

43. Van Dongen $M$, Savage ND, Jordanova ES, Briaire-de Bruijn IH, Walburg KV, Ottenhoff TH, Hogendoorn PC, van der Burg SH, Gelderblom H, van Hall T.. Anti-inflammatory M2 type macrophages characterize metastasized and tyrosine kinase inhibitor-treated gastrointestinal stromal tumors. Int J Cancer 2010;127(4):899-909. doi: 10.1002/ijc. 25113 